\title{
Research on the Key Technology of Augmented Reality Technology in the Application and Development of Children's Plant Science Popularization Education
}

\author{
Yuyi Ding ${ }^{1,}$, Jinghua Han ${ }^{1, b^{*}}$ and Qi Cao ${ }^{1, c}$ \\ ${ }^{1}$ Beijing Forestry University, Beijing, China

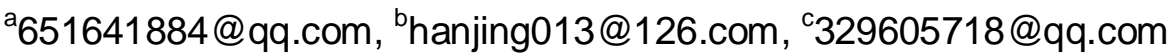

\begin{abstract}
Keywords: Augmented reality; Children's education; Plant science popularization
\end{abstract}
\begin{abstract}
In recent years, the development and application of augmented reality technology has become a newly emerging and popular research field. The multi-dimensional display, virtual-actual combination, real-time interaction and other characteristic of the technology meet the demands of science popularization education. It has a unique advantage in the information display and has laid a good foundation for the research and development of innovative children's science popularization education model. This paper, taking the self-developed children's plant science popularization education application as an example, analyzes and researches four technical contents: the augmented reality core technology, the real-time rendering technology, the interaction technology and the 3D model design, and explores the application value of augmented reality technology in the field of education.
\end{abstract}

\section{Introduction}

The Augmented Reality Technology is an important branch of Virtual Reality Technology [1]. On the basis of virtual reality technology, augmented reality technology uses computer graphics technology and visualization technology [2] to superimpose virtual images generated by computer operations to real pictures. Finally, the virtual images are combined with the real pictures, and the technology that real world scene is enhanced or expanded by using the additional information generated by the computer[3]. They complement each other, using the display device to present a "virtual in the real" visual effect for the user, which can not only present virtual objects in the real 3D space environment, but also present the non-geometric information related to real objects [4]. It apples the newly emerging and popular augmented reality technology in the field of children's science popularization education in recent years, and it is a helpful exploration and research on the mode of children's science popularization education.

The author's self-developed children's plant science popularization education is made up of App on intelligent mobile terminal and object AR plant card, developed by Unity 3D engine, and takes Vuforia as the development tool of augmented reality technology. By combining the rich plant science popularization knowledge with the interesting augmented reality technology, the goal of teaching goes together with pleasure is achieved.

\section{Methods and Key Technologies}

Augmented reality core technology. The augmented reality technology applied to augmented reality children's plant education products includes three core technologies: Image Target scanning and identification, Track able Behavior customization, and camera auto- focus.

Image Target scanning and identification: this technology is the key technology to augment the reality scanning and identification marker as well as generate the corresponding 3D model, use the camera to capture and identify the marker, the 3D plant model is generated and displayed with the aid of the pre-set relationship between 3D plant model and the spatial location of marker. Taking the Vuforia development tool from American PTC company as an example, the development tool can run in the Unity 3D engine, use the Unity 3D engine to bind the 3D plant model with the spatial 
location of marker, and complete the 3D registration of marker with by use of AR Camera component of Vuforia, and realize Image Target scanning and identification (Figure 1).

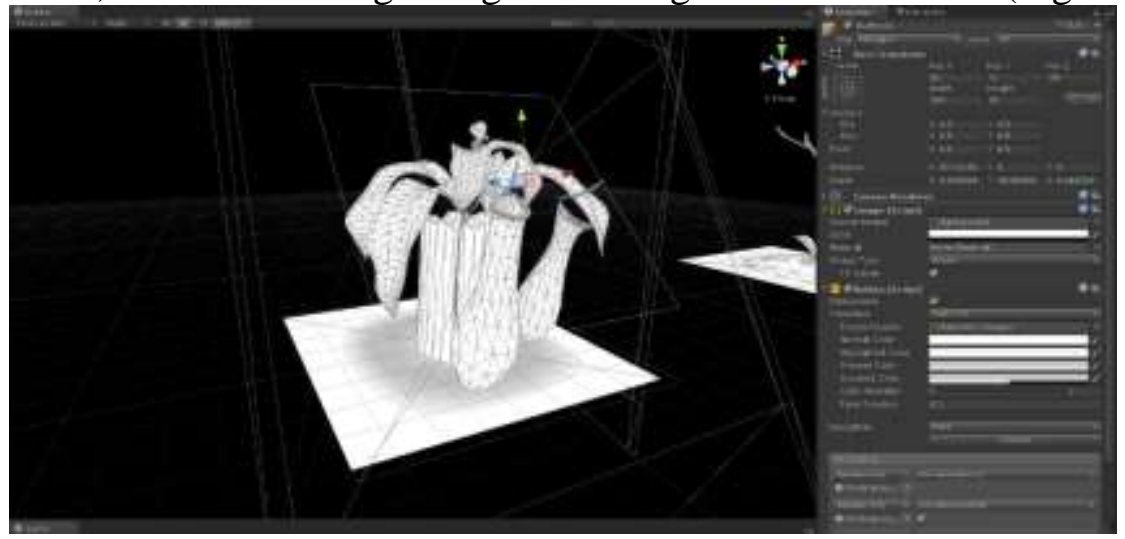

Figure 1. The binding of 3D plant model with spatial location of marker

Track able Behavior customization: Vuforia development tools only provide two default tracing behaviors: creating models and destroying models for developers, far from meeting the actual needs of augmented reality children's plant science popularization. In order to realize the correct identification of animation, sound and interaction interface by product App, On Tracking Found and On Tracking Lost program statements need to be edited. Using the for each statement, the creating and destroying behaviors for Animation component, Audio Source component, and Canvas component are edited, so as to create correct 3D plant model, animation, sound effect and interaction interface after scanning and identifying the marker.

Camera auto-focus: in order to ensure the identification speed and shooting clarity of augmented reality scanning, Vuforia development tools need to use the camera auto-focus function. As the camera's hardware specifications of different types of intelligence terminals are generally very different, the hardware interface statement of corresponding used camera also has the corresponding differences. In order to facilitate unified control and reduce the circulation of similar type program statements, Vuforia Camera Device Instance Set Focus Mode statement built in Vuforia development tool can be used to obtain all the auto-focus functions control authority of the camera hardware with auto-focus function.

Real-time rendering special effect technology. Real-time rendering is a special effect technology to render the content of screen as a whole. The reasonable use of real-time rendering within the range of hardware function can effectively improve the quality of 3D real-time rendering, making the picture softer, natural fresh and bright. The graphical and image interface supported by hardware platform should be determined before applying the real-time rendering effect. For example, the PC terminal supports DirectX and OpenGL graphical and image interface, the mainstream Android terminal generally supports the OpenGL graphical and image interface, and the iOS terminal supports the OpenGL and Metal graphical and image interface. In order to facilitate the development of multi platform and save the cost of development, the OpenGL graphical and image interface with good compatibility can be used.

Taking Unity 3D as an example, the real-time rendering special effect is applied to the Camera component and the Camera component is embedded with different Image Effects, that is, the application of real-time rendering special effect can be realized. Some Image Effects should be adjusted according to the actual requirements, for example, Bloom is preset with two rendering levels of Cheap and High, and with two rendering modes of Basic and Complex, which is used to correspond to different hardware configurations. In addition to using the preset parameter to adjust the content, Unity 3D also supports user customized editing scripts, using the public statement to call the corresponding rendering effects, such as public varflareColor : Color $=$ Color $(0.4 \mathrm{f}, 0.4 \mathrm{f}$, $0.8 \mathrm{f}, 0.75 \mathrm{f}$ ), and this statement can realize the bright display effect of designated color (Figure 2). 


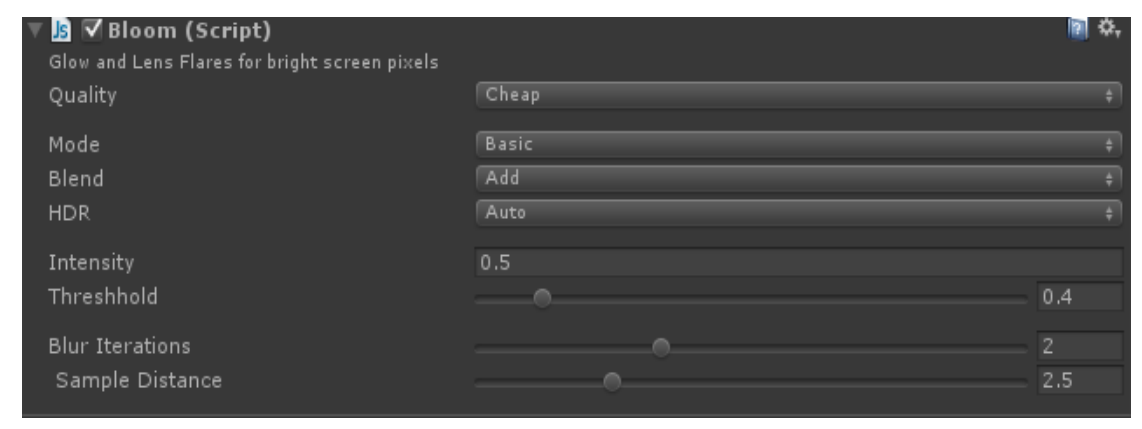

Figure 2. Bloom rendering special effects and rendering parameters

Interaction process design. Children's logic thinking ability is still at the stage of growth and development, and they still do not possess complex and rigorous logic thinking ability. Too complex interaction logic will make children encounter excessive obstacles in the process of learning and eliminate children's learning enthusiasm, so the concise and intuitive interaction logic design is very necessary. In the process of interaction design, it needs to avoid too many levels in the same level menu, and the logic level should take horizontal arrangement as the main part, and reduce the vertical recursion arrangement. After clicking the corresponding interaction button, children can enter directly to the interface they want to enter. For example, when the user clicks the "start" button, that is, entering directly into the main application, the advanced function is displayed directly on the interface of main application, in order to facilitate the use of children. The interaction logic flow chart of software is shown in Figure 3.

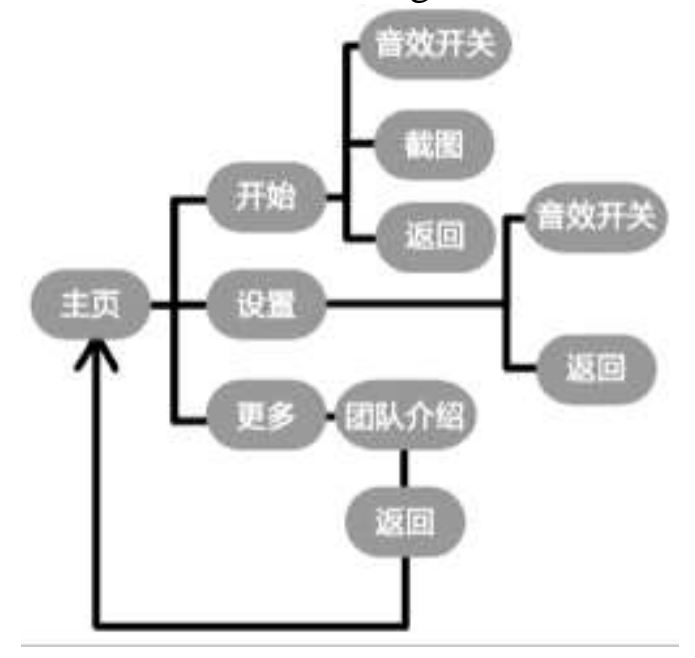

Figure 3. Interaction logic flow chart

Interaction script. Interaction script mainly realizes the function of identifying screen click, triggering scene switching, triggering event, creating and outputting information. In script code writing, it is necessary to pay attention to the rigorous and concise statement logic, and avoid the vicious Bug caused by the memory overflow that is caused by the implementation of useless loop statement and it will result in the program collapsing. Taking the trigger scene switching as an example, the public void Click statement is used to realize the identification of Button touch, and the scene switching is realized by using the Application. LoadLevel statement. There is not only the input behavior, but also the output behavior in the interaction process, such as it needs to generate a screenshot and be named by the current system time, and System. DateTime now = new System. DateTime can be used to redefine the current system time so that the system time can correctly identified, and the screenshot is generated by usingApplication. CaptureScreenshot(filename,0) statement and the screenshot is named as the current system time.

3D space interaction triggering technology. The interaction mode of AR technology application in the range of present study is mainly based on screen touch, but it is different from the 
traditional plane interaction. In the AR technology, there is a combination of $2 \mathrm{D}$ and 3D information with the real depth relationship.

The traditional plane interaction triggering mechanism will deviate by the depth perspective relation in the picture with depth relationship, and its principle can be analogized to the picture in $3 \mathrm{D}$ coordinate system projecting in the 2D coordinate system (Figure 4). In order to solve this problem, the triggering conditions of each interaction trigger component need to be divided into group for linkage and IK calculation. According to the change of distance between the scanning of hardware device and shooting angle, the interaction component will make corresponding change with the perspective law in the 3D space.

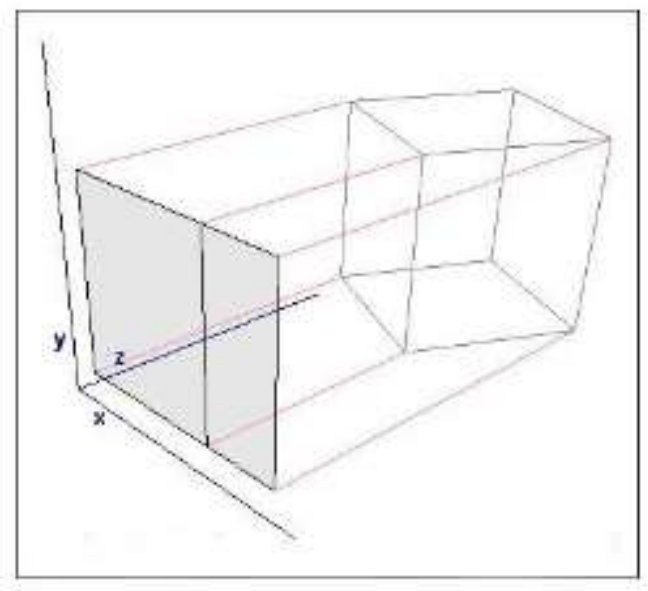

Figure 4. Object in 3D coordinate system projecting in 2D coordinate system

Plant 3D model design. The 3D visual display in the augmented reality technology is different from the general 3D visual display. It does not rely on the interaction interface of camera and the interaction input equipment, and uses the camera of hardware equipment to take the picture directly. It is easy to operate and is suitable for children to use.

Plant modeling process mainly uses 3D modeling software, such as Maya, 3ds Max, Zbrush and so on. Using polygon modeling to build the low model of plant model, the average distribution of vertex is made to ensure that the distance between point and point is moderate, the surface of vertex is four sides, and the triangle can appear properly, surface and surface are directly connected to form the topology structure, in order to avoid the interspersed and overlapped model surface.

The low model of plant 3D model is developed by UV, and Diffuse mapping is drawn in Photoshop software according to UV information. The mapping formats are generally JPEG (Joint Photographic Expert Group), PNG (Portable Network Graphics), TIFF (Tag Image File Format), TGA (Tagged Graphics). JPEG format file has high compression rate, small file volume, no Alpha channel, and it can be mainly used in Diffuse material; PNG format file has moderate-quality picture, small file volume and Alpha channel, it can be used for Alpha material; TIFF, TGA format files have high color reduction, Alpha channel, large file size, and it is suitable for Alpha Blended, Specula and other advanced materials. The picture format selection is determined by the application program operation platform and the mapping material. The application in the PC terminal can select the TIFF and TGA files with good generality, high quality and larger volume. The application of mobile terminal can select the JPEG and PNG format files with smaller volume and faster call rate.

In order to present the texture of plant models, Normal Map should be baked for the model. Taking Zbrush as an example, Normal Map's baking sampling follows the vertex coloring rule, and the higher the number of vertices, the higher the sampling accuracy. The sculpture of plant model is completed at high subdivision and high vertex number level, then return to the minimum subdivision and minimum vertex number level, the Normal Map is made by using the Normal Map baking tool in Zbrush, and it is finally given to the Shader of Bumped Diffuse. 
Taking Hypershade in Maya software as an example, the Diffuse Map and Normal Map are connected to the 3D model in the form of node. In order to reduce the hardware performance consumption, the non-transparent channel materials adopts Lamber Shader (Figure 5).

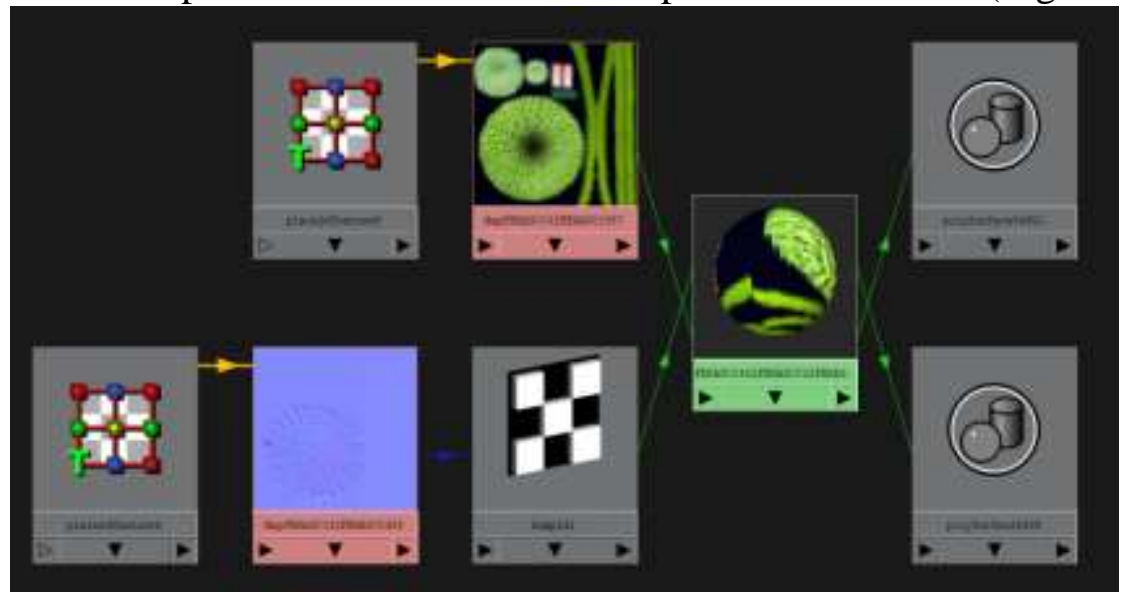

Figure 5. Hypershade node connection figure

\section{Conclusion}

The application of augmented reality children's plant education has met the psychological and physical behavior characteristics of children, with the interaction design and animation content conforming to plant characteristics, rich and interesting. And it contains plant knowledge, makes full use of unique interaction and entertainment of augmented reality technology, brings children into a vivid and lifelike plant world, and offers them a new learning cognitive experience.

Augmented reality technology has integrated a variety of media methods and a number of technical contents, can create various interesting display and interaction and bring users unprecedented use experience, meanwhile, it has opened a new road for the development of display model and interaction mode. Combining with the application and development of this technology in children's science popularization is a helpful attempt for augmented reality technology in the field of application and it also guides the new direction for the exploration of children's education mode.

\section{Acknowledgments}

This work is supported by the Fundamental Research Funds for the Central Universities (2015ZCQ-YS-02) and Beijing Higher Education Young Elite Teacher Project (YETP0785).

\section{References}

[1] Lei Jing. Application of augmented reality technology in teaching, J. China Educational Technique \& Equipment, Vol. 35 (2011), p.7-8.

[2] Lin Liang, Yang Ke, Wang Yongtian. Research on key technology of mobile augmented reality system, J. Chinese Journal of Image and Graphics, Vol. 14 (2009) No.3, p.560-564.

[3] Chen Jing, Shi Qi, Wang Yongtian. Augmented reality technology and its application, J. Computer Engineering and Application, Vol. 37 (2001) No.21, p.55-57.

[4] Azuma R, Baillot Y, Behringer R, etal. Recent advances in augmented reality, J. IEEE Computer Graphics and Applications, Vol. 21 (2001) No.6, p.34-47. 